\title{
ORÇAMENTO BASE ZERO: UM ESTUDO NAS UNIVERSIDADES CATARINENSES
}

\author{
ZERO-BASED BUDGETING: A STUDY IN UNIVERSITIES CATARINENSES
}

\author{
Adriano Sérgio Da Cunha \\ Universidade do Sul de Santa Catarina - Unisul \\ Thiago Coelho Soares \\ Universidade do Sul de Santa Catarina - Unisul \\ Carlos Rogério Montenegro De Lima \\ Universidade do Sul de Santa Catarina - Unisul
}

\section{RESUMO}

Este artigo tem por objetivo analisar a aplicação orçamentária nas universidades de Santa Cataria. Assim, o problema de pesquisa pode ser definido como "O Orçamento Base Zero é utilizado como ferramenta gerencial pelas Universidades do Estado de Santa Catarina?". Para alcançar este objetivo foi elaborada uma pesquisa qualitativa de caráter descritivo nas vinte e três universidades credenciadas pelo Ministério da Educação para atuar no território catarinense. O Orçamento Base Zero não utiliza o orçamento anterior ou despesas para elaborar um novo período orçamentário, uma vez que as circunstâncias e as finanças da organização podem ter mudado. Ao elaborar um orçamento a partir de uma base zero, as despesas devem ser justificadas. Isto ajuda a controlar os gastos, pois o orçamento é montado a partir de zero, em vez de ser construído sobre valores que foram gastos durante o período anterior, já que, na maioria das vezes, esses números já estão ultrapassados e possivelmente distorcidos. Nesta pesquisa será possível identificar se a utilização do orçamento de base zero pode auxiliar no processo de gestão das instituições no curto prazo, podendo se configurar como um diferencial competitivo.

Palavras-Chave: Orçamento. Orçamento base zero. Universidades.

\begin{abstract}
This article aims to analyze the bugeting implementation in the universities of Santa Catarina. Thus, the research problem can be defined as "Zero-Based Budgeting is used as a management tool by the University of Santa Catarina?". To achieve this objective was drawn from a qualitative descriptive character in the twenty-three universities accredited by the Ministry of Education to serve on the territory of their state. The Zero-Based Budgeting does not use the previous budgeting or expense to draft a new budgeting period, as circumstances and finances of the organization may have changed. In preparing a budgeting from a zero based, the expenditure must be justified. This helps to control spending because the budgeting is assembled from scratch, instead of being built on values that were spent during the previous period, since in most cases, these figures are over and possibly distorted. This research will help to identify whether the use of Zero-Based Budgeting can help in the management of the institutions in the short term and can be configured as a competitive differentiator.
\end{abstract}

Keywords: Budgeting. Zero-based budgeting. Universities. 


\section{INTRODUÇÃO}

O cenário da educação superior brasileira oportuniza as instituições a se moldarem ao acirramento do mercado por meio de alterações diretivas e possivelmente na natureza estratégica da instituição. 0 planejamento orçamentário pode subsidiar informações necessárias para a instituição renovar as políticas de seu negócio, fornecendo informações que podem auxiliar a definição do posicionamento competitivo, que promoverá a sustentabilidade em longo prazo da instituição.

O planejamento estratégico da organização é o primeiro passo para elaborar o orçamento. 0 planejamento direciona o que queremos de fato fazer e o orçamento nos diz quanto vamos gastar para realizar o planejamento. Para se ter um bom orçamento necessita-se que o planejamento esteja muito bem feito, minimizando as possibilidades de erros e falhas.

Assim, o orçamento é derivado dos dados apurados no planejamento. Confeccionado o orçamento, simulam-se os resultados e depois disso pode-se optar pelos cortes orçamentários, optando pela exclusão de planos não econômicos ou de pouco retorno ou pela exclusão de determinadas despesas. Na conclusão do orçamento chega-se a uma das etapas mais importantes, que se refere ao acompanhamento e gerenciamento do orçamento. (BATISTA, 2007)

O Orçamento Base Zero (OBZ) é uma abordagem orçamentária desenvolvida nos Estados Unidos da América pela Texas Instruments Inc. durante o ano de 1969. Foi adotado pelo estado de Geórgia no governo Jimmy Carter com vistas ao ano fiscal de 1973. Suas principais características: análise, revisão e avaliação de todas as despesas propostas e não apenas das solicitações que ultrapassam o nível de gasto já existente; todos os programas devem ser justificados cada vez que se inicia um novo ciclo orçamentário. (RAZA, 2010)

Para se elaborar o OBZ é necessário estudar as despesas uma a uma, para identificar os possíveis excessos ou carências nos gastos de cada item. É necessário analisar cada despesa, como ela é feita, quando é feita, elaborar premissas e designar responsáveis pelo gerenciamento da despesa. O OBZ é um "instrumento vivo" de gerenciamento que se adapta às novas tendências situacionais ou mercadológicas tendo como componentes básicos quatro princípios: planejamento, orçamento, implantação e controle (BATISTA, 2007).

Desta forma, este trabalho teve por finalidade analisar a aplicação orçamentária nas Universidades de Santa Cataria. Assim, o problema de pesquisa pode ser definido como "O Orçamento Base Zero é utilizado como ferramenta gerencial pelas Universidades do Estado de Santa Catarina?"

Esta pesquisa teve caráter qualitativo. A pesquisa qualitativa, de acordo com Triviños (1994), permite analisar os aspectos implícitos ao desenvolvimento das práticas organizacionais e a interação entre seus integrantes. É a técnica mais apropriada para compreender o fenômeno no contexto onde está inserida e do qual é parte, pois possibilita analisá-lo numa perspectiva integrada. $\mathrm{O}$ autor ainda enfatiza que a pesquisa qualitativa possibilita o uso da imaginação e da criatividade do pesquisador e da realidade sobre o tema escolhido, explorando os mais diversos enfoques para o enriquecimento do seu estudo.

A pesquisa quanto aos fins pode ser considerada descritiva. É descritiva, pois descreve as informações obtidas por meio da pesquisa documental. Para Churchill (1987) pesquisa descritiva objetiva conhecer e interpretar a realidade sem nela interferir para modificá-la. A pesquisa descritiva expõe as características de determinada população ou de determinado 
fenômeno, mas não tem o compromisso de explicar os fenômenos que descreve, embora sirva de base para tal explicação.

A pesquisa procurou abranger um grande número de publicações nacionais e internacionais, apesar das poucas citações sobre o assunto. Quanto às publicações nacionais, sua pesquisa foi realizada através da visita a várias bibliotecas e também pela busca de periódicos junto ao portal Qualis. O processo de busca foi feito através das palavras chaves: orçamento, orçamento base zero, e pela busca de autores cuja citação já ocorreu em publicações nacionais; além da leitura preliminar dos resumos para verificar a pertinência temática. Quanto aos artigos internacionais foi utilizado a base da Ebsco para a procura. No tocante a produção científica de 1995 a 2006, conforme Leite et al (2008) é possível identificar apenas uma dissertação sobre orçamento base zero, em 2002: "Planejamento e Orçamento Base-zero: as possibilidades utilização desse instrumento para melhoria gerencial da Universidade Federal de Mato Grosso".

\section{REVISÃO DA LITERATURA}

$\mathrm{Na}$ revisão da literatura serão abordados os termos instituições de ensino superior, orçamento e orçamento base zero.

\section{INSTITUIÇÕES DE ENSINO SUPERIOR}

Colossi (2001) afirma que o reconhecimento crescente da administração universitária é resultante de uma complexidade cada vez maior da educação superior. Isso porque, as transformações sociais e culturais acarretam mudanças significativas no processo educacional, e modificam até mesmo o significado da educação. Por outro lado, as transformações no âmbito das organizações complexas exigem novas formas de gestão, maior flexibilidade organizacional, com sistemas decisórios mais participativos.

Segundo Teixeira (1964), as universidades têm uma longa história, durante a qual passaram por transformações. Das grandes funções universitárias, a primeira é a formação profissional. A segunda grande função corresponde ao alargamento da mente humana, que o contato com o saber e a sua busca produzem nos que freqüentam a universidade. A terceira função é a de desenvolver o saber humano. A quarta e última função seria de transmissora de uma cultura comum.

A Universidade é, conforme Melo (2002), uma das instituições mais importantes da sociedade no momento atual, isso porque se considera a competitividade das economias globalizadas e o processo de inovação e mudanças contínuas nas organizações. Assim, insere-se na discussão das funções universitárias uma corrente em que o processo econômico e social dos países depende, invariavelmente, da ação direta desse tipo de instituição, destacando-se como um valioso patrimônio no exercício de suas funções de ensino, pesquisa e extensão.

Segundo Morgan (2003), nas universidades brasileiras, principalmente as públicas, são executadas, além do ensino e extensão, atividades relacionadas à pesquisa. Os recursos utilizados nas pesquisas são oriundos de financiamentos de organizações que, previamente, aprovam um projeto, no qual conste o orçamento financeiro necessário para o desenvolvimento do estudo.

Conforme Amaral (2003), os elevados gastos destinados ao ensino superior em comparação aos outros níveis de ensino, e a divulgação constante de elevados valores para o custo dos alunos das IFES, foram dois fatos que provocaram desgaste das instituições de ensino 
superior perante a população. Ainda conforme o autor, muitas comparações entre os gastos públicos brasileiros com o estudante do ensino superior e com os estudantes dos outros níveis de ensino foram realizadas, essas discussões não levavam em conta que o ensino superior, que é caracterizado pela indissociabilidade entre ensino, pesquisa e extensão, é muito mais caro que os outros níveis de ensino.

\section{ORÇAMENTO}

Os sistemas orçamentários considerados eficazes viabilizam um sistema de planejamento e controle. 0 planejamento corresponde à fixação de objetivos e elaboração de orçamentos para alcançá-los. Porém, planejar se torna insipiente caso não haja acompanhamento. No processo de acompanhamento, o controle tem a finalidade de assegurar que os objetivos e metas planejadas e implementadas na fase de execução sejam atingidos. (ALMEIDA et al, 2009).

King, Clarkson e Wallace (2010) apresentam evidências que ligam as características básicas de saúde empresarial, as práticas de orçamento e o desempenho das empresas. Com base numa amostra de 144 respostas de um questionário junto aos membros da Associação Australiana de Práticas Gerenciais (AAPM), descobriu-se que os fatores identificados pela pesquisa de contingência são úteis para prever as práticas de um orçamento empresarial. Especificamente, descobriu-se que a adoção de orçamentos por escrito está relacionada ao tamanho e estrutura, e para as empresas com orçamentos por escrito, a extensão do uso está relacionado à estrutura do negócio, estratégia e percepção de incerteza ambiental. Finalmente, encontrou-se evidências de uma relação entre a prática do orçamento e desempenho. Aqui, inicialmente encontra-se um desempenho do negócio a ser positivamente associada com o uso dos orçamentos por escrito.

O orçamento historicamente desempenhou um papel fundamental no controle da gestão, no entanto, recentemente tem sido objeto de crítica considerável e debate. Alguns argumentam sobre os problemas causados pelo maneira como são utilizados os orçamentos, enquanto outros argumentam que os processos orçamentários são fundamentalmente falhos.

Outros têm chamado a atenção para uma análise sistemática destas questões contra a evidência empírica. Em geral, na maioria das empresas os orçamentos continuam a ser usados para fins de controle e são percebidos como valor agregado. Enquanto existem problemas com os orçamentos, as organizações estão se adaptando a sua utilização para dar conta desses problemas ao invés de abandonar completamente os orçamentos. (BENNOUNA; MEREDITH; MARCHANT, 2010)

A prática do orçamento com base histórica predomina entre uma variedade de bases orçamentárias. Além disto, a elaboração orçamentária é muito limitada por orçamentos anteriores e pré-limites orçamentais, tais como custos orçamentários significativos, que podem ser economizados através da agregação de pequenos contratos em pacotes maiores de contratos (LAI, 2010).

Em meio a tantas incertezas de hoje, a maioria das empresas estão cortando gastos indiscriminadamente. 0 processo de orçamento típico não é, contudo, desenhado para os gestores repensarem seus modelos de negócio se persistir a recessão da economia ou mudanças de um modo fundamental. Pelo contrário, muitos orçamentos atuais estão ancorados em orçamentos anteriores, com mudanças incrementais para ajustar para a inflação ou tendências específicas do produto (AKTEN; GIORDANO; SCHEIFFELE, 2009). 


\section{ORÇAMENTO BASE ZERO}

O Orçamento Base Zero tornou-se opção de inúmeras empresas na tentativa de melhorar seu processo orçamentário em virtude das condições da atividade empresarial em constantes mudanças. As empresas vem sendo afetadas por fatores internos e externos, tais como: acionistas mais exigentes; clientes mais exigentes; escassez de mão-de-obra qualificada; necessidade de inovação; preços menores e qualidade superior; e maiores exigências por ética e responsabilidade social (LUNKES, 2003).

O orçamento base zero é o processo orçamentário, onde cada item tem de se rejustificar para obter aprovação. O OBZ baseia-se em um conceito claro de uma empresa de objetivos a longo prazo, metas específicas de curto prazo e os recursos financeiros disponíveis para ser bem sucedida. Além disso, ele se concentra nas atividades das despesas ao invés de departamento ou tipo de item do orçamento (MATURI, 2009).

"O objetivo do Orçamento Base Zero é o de viver de acordo com os recursos disponíveis; e corresponde a um meio de analisar, reestruturar e eliminar despesas, programas e projetos não econômicos" (PREMCHAND, 1998).

O Orçamento Base Zero foi desenvolvido durante o ambiente inflacionário de meados da década de 1970 para evitar esta armadilha. O OBZ começa o processo todo a partir do zero, assumindo os pontos finais diferentes para diferentes indústrias e empresas, tais como trinta por cento menor no mercado global. Gastos operacionais e despesas de capital são, então, priorizados de acordo com o seu alinhamento com a estratégia da empresa e seus retornos esperados sobre o investimento (AKTEN; GIORDANO; SCHEIFFELE, 2009).

O Grupo Pão de Açúcar implantou a ferramenta de gestão Orçamento Base Zero. Esta ferramenta é amplamente utilizada pela AmBev - famosa por seu modelo gerencial agressivo e totalmente focado em resultados. Após a implantação da solução Dígitro, a Nova Iguaçu Distribuidora de Bebidas passou a usufruir de benefícios de ordem financeira, com o controle total dos gastos e a estabilidade nas despesas de telefonia - resultado obtido principalmente com a utilização do Rota Econômica e do STAB. Tal redução de custos veio de encontro com as metas estabelecidas no "Centro de Custo OBZ AmBev": Orçamento Base Zero - indicador utilizado por todas as distribuidoras AmBev. Outros benefícios que vem sendo percebidos são de ordem tecnológica, com a integração perfeita da solução Dígitro, de maneira estável e segura, com o perfil tecnológico da empresa e seguindo o padrão de qualidade exigido pela AmBev. (RAZA, 2010)

Um player europeu das telecomunicações iniciou um processo de orçamento base zero desagregando seus gastos em unidades de decisão lógica, abordando diferentes tipos de despesas, tais como novas despesas de capital (por exemplo, construindo uma rede de terceira geração) ou de manutenção. Cada unidade de decisão de gastos de capital (tais como aquelas para satisfazer as exigências de licença ou de crescimento de uma cidade orientada) foi classificada como "manter", "discutir", ou "cortar". Finalmente, os executivos discutiam a prioridade de cada despesa de capital em seus retornos financeiros e o alinhamento com a estratégia da empresa. Depois de apenas algumas interações, a empresa atingiu o seu objetivo relacionado às despesas de capital - uma redução de 20 por cento, apoiado o investimento no crescimento futuro (AKTEN; GIORDANO; SCHEIFFELE, 2009).

Costa, Moritz e Machado (2007) concluem que o Orçamento Base Zero não é simplesmente uma ferramenta de gestão empresarial que tem por intenção a redução de custos e gerar 
economia para as empresas, mas sim uma ferramenta que promove o desenvolvimento das melhores práticas para elaborar as suas atividades, assim como para otimizar os recursos financeiros.

\section{ANÁLISE DOS DADOS}

Em Santa Catarina, a demanda reprimida existente a partir da década de sessenta proporcionou considerável crescimento do número de instituições de ensino superior no estado. Assim, as instituições de ensino superior, considerando suas fragilidades individuais, buscaram se unir em torno de um órgão com as atribuições de planejar, articular e coordenar integradamente suas ações. Em 1974, criou-se a ACAFE - Associação Catarinense das Fundações Educacionais -, inicialmente congregando as 16 instituições existentes. A ACAFE é uma entidade sem fins lucrativos, com a missão de promover a integração dos esforços de consolidação das instituições de ensino superior por elas mantidas, de executar atividades de suporte técnico-operacional e de representá-las junto a órgãos dos Governos Estadual e Federal. Hoje são mais de uma centena de instituições de ensino superior atuando no estado, e o número cresce ano após ano.

Com o acréscimo no número de IES no estado, criou-se um cenário mais competitivo. Assim, em mercados mais competitivos existe um recálculo dos custos, com o intuito de colocar à disposição de seus clientes produtos ou serviços com alta qualidade e com preço justo. Nesse mesmo sentido, as universidades desenvolveram um conjunto complexo de atividades, estas propiciam a obtenção dos mais variados tipos de custos, tais como o custo por aluno, por estudante, custo da pesquisa, custo da extensão, custo do hospital universitário, custo das atividades administrativas entre outros. Assim, o orçamento passa a ser uma ferramenta gerencial que pode auxiliar no processo de tomada de decisão.

Um orçamento expressa quantitativamente um plano de ação e auxilia na coordenação e implementação de um plano. Orçar corresponde a processar os dados constantes do sistema de informação contábil, inserindo informações previstas e considerando alterações já definidas para o próximo período. 0 orçamento tem por obrigação reunir diversos objetivos empresariais na busca da expressão do plano e controle de resultados. Talvez o ponto crucial seja o processo de estabelecer e coordenar objetivos para todas as áreas da instituição, de maneira que todos trabalhem sinergicamente em busca de resultados positivos. Assim, o orçamento pode se tornar uma ferramenta que auxilie os gestores das instituições de ensino superior a planejar e controlar os gastos.

Diante da necessidade de melhorar o desempenho, diversas empresas estão buscando alterar seu elemento central do sistema de gestão: o orçamento. O Orçamento Base Zero tornou-se opção de inúmeras empresas na tentativa de melhorar seu processo orçamentário.(LUNKES, 2003). E isto que vamos verificar na análise das Universidades que atuam no território catarinense.

A partir de pesquisa feita no e-MEC identificou-se as universidades que estão credenciadas para atuar em Santa Catarina com ensino presencial. Encontrou-se vinte e três universidades. 
Quadro 1: Universidades Credenciadas para atuar em Santa Catarina

\begin{tabular}{|l|l|l|}
\hline UNIARP & UNIVERSIDADE ALTO VALE DO RIO DO PEIXE & PRIVADA \\
\hline UNIDERP & UNIVERSIDADE ANHANGUERA - UNIDERP & PRIVADA \\
\hline UAM & UNIVERSIDADE ANHEMBI MORUMBI & PRIVADA \\
\hline UCB & UNIVERSIDADE CASTELO BRANCO & PRIVADA \\
\hline UNOCHAPECO & UNIVERSIDADE COMUNITÁRIA REGIONAL DE CHAPECÓ & PÚBLICA \\
\hline UNIVILLE & UNIVERSIDADE DA REGIÃO DE JOINVILLE & PRIVADA \\
\hline UNC & UNIVERSIDADE DO CONTESTADO & PRIVADA \\
\hline UNESC & UNIVERSIDADE DO EXTREMO SUL CATARINENSE & PÚBLICA \\
\hline UNOESC & UNIVERSIDADE DO OESTE DE SANTA CATARINA & PÚBLICA \\
\hline UNIPLAC & UNIVERSIDADE DO PLANALTO CATARINENSE & PRIVADA \\
\hline UNISUL & UNIVERSIDADE DO SUL DE SANTA CATARINA & PRIVADA \\
\hline UNITINS & UNIVERSIDADE DO TOCANTINS & PÚBLICA \\
\hline UNIVALI & UNIVERSIDADE DO VALE DO ITAJAÍ & PRIVADA \\
\hline UNISINOS & UNIVERSIDADE DO VALE DO RIO DOS SINOS & PRIVADA \\
\hline UNESA & UNIVERSIDADE ESTÁCIO DE SÁ & PRIVADA \\
\hline UFSC & UNIVERSIDADE FEDERAL DE SANTA CATARINA & PÚBLICA \\
\hline ULBRA & UNIVERSIDADE LUTERANA DO BRASIL & PRIVADA \\
\hline UNIP & UNIVERSIDADE PAULISTA & PRIVADA \\
\hline UNP & UNIVERSIDADE POTIGUAR & PRIVADA \\
\hline FURB & UNIVERSIDADE REGIONAL DE BLUMENAU & PUACA \\
\hline UNIVERSO & UNIVERSIDADE SALGADO DE OLIVEIRA & \\
\hline UNIFACS & UNIVERSIDADE SALVADOR & FUNDAÇÃO UNIVERSIDADE DO ESTADO DE SANTA CATARINA \\
\hline UDESC & FADA & PUACA \\
\hline
\end{tabular}

Fonte: E-MEC

Com as informações do quadro 1 foi possível acessar o site das instituições e verificar a existência de relatórios que permitissem a averiguação da utilização do Orçamento Base Zero como ferramenta de apoio a tomada de decisões.

Das vinte e três universidades que tiveram seus sites analisados, dez não tiveram informações gerenciais relevantes encontradas: Univali, Uniarp, Uniderp, Unochapecó, Unitins, Unisinos, Unip, Universo, Unifacs e Udesc. Outras instituições apresentaram informações com acesso apenas para comunidade interna: Furb (Avaliação institucional) e Univille (planejamento estratégico). Outras cinco instituições apresentavam relatórios, mas que não poderiam contribuir para o estudo: Unoesc (Estatuto), Uam (Avaliação institucional 2006, 2007, 2008), Ucb (Avaliação institucional), Unesc (balanço social 2004, 2008) e Ulbra (Ulbra em dados e balanço social). Já seis instituições apresentaram relatórios, em seus sites, que foram úteis para o alcance dos objetivos: Unisul (Relatório de Gestão 2001-2009 e orçamento 2009 e 2010), UnC (Demonstrativos Contábeis 2006, 2007, Plano de Desenvolvimento Integrado), Uniplac (demonstrativos financeiros, balanço social), Unesa (empresa de capital aberto, com 
todas as informações), UFSC (boletim de dados) e Unp (Plano anual de trabalho e projeto pedagógico institucional).

Desta forma, empiricamente, estabeleceu-se que apenas as últimas seis universidades poderiam ser alvo mais aprofundado deste estudo. Nos relatórios estudados, nenhum deles menciona o OBZ como ferramenta utilizada ou a ser implantada na instituição.

A UnC em seu plano de desenvolvimento integrado não apresenta o orçamento da instituição, mas relata a utilização de orçamento setorizado.

Na Unisul a elaboração da proposta orçamentária obedece às diretrizes estratégicas definidas pela Administração Superior da Universidade. As informações são apresentas como orçamento mensal consolidado para a instituição.

A Unp pretende adotar o orçamento como base de gestão e tomada de decisões de sustentabilidade.

A UFSC segue a regra orçamentária definida pela União e fiscalizada pelo TCU, por se tratar de uma instituição de ensino superior federal.

A Uniplac, em cumprimento as disposições legais e estatutárias, apresenta para a devida apreciação o Balanço Patrimonial e Demonstrativos Financeiros. Porém, os dados mais recentes são do encerramento de 2007. Não faz menção ao orçamento, apenas as necessidades legais de publicação de relatórios contábeis.

A Unesa, em julho de 2008, aderiu ao Novo Mercado. Neste sentido, a Companhia está vinculada à arbitragem perante a Câmara de Arbitragem do Mercado, conforme estipulado em seu Estatuto Social. A Companhia possui um Conselho Fiscal formado por três membros independentes, sendo um deles apontado pelos acionistas minoritários. Embora apresente todas as informações necessárias para uma companhia de capital aberto, as informações sobre orçamento são nulas.

O Orçamento Base Zero é uma previsão orçamentária projetada, na qual não se leva em consideração o que ocorreu nos anos anteriores. Porém, nenhuma instituição pesquisada deixou claro a utilização de tal ferramenta, o que pode ser considerado danoso para as instituições de ensino, principalmente pelas constantes alterações do cenário educacional.

De acordo com dados divulgados pelo IBGE, em sete anos o contingente médio anual da população desocupada no Brasil caiu 28,2\%, o que corresponde a menos 736 mil pessoas desempregadas. Sendo o setor de ensino superior privado no Brasil composto, em sua maioria, por adultos inseridos no mercado de trabalho, acredita-se que os números de crescimento da economia e do nível de emprego brasileiros tenham direta influência na demanda pelos serviços de educação. (ESTÁCIO DE SÁ, 2010).

No quadro 2, observa-se o crescimento do número de matrículas e o percentual da população matriculado. 
Quadro 2: Alunos matriculados no ensino superior

\begin{tabular}{|c|c|c|c|}
\hline Ano & População de 18 a 24 anos & Matrículas Brutas & \% da população matriculada \\
\hline 2000 & 23.365 .185 & 2.694 .245 & 11,53 \\
\hline 2001 & 22.940 .218 & 3.030 .754 & 13,21 \\
\hline 2002 & 23.098 .462 & 3.479 .913 & 15,06 \\
\hline 2003 & 23.371 .702 & 3.887 .022 & 16.63 \\
\hline 2004 & 24.072 .318 & 4.163 .733 & 16,63 \\
\hline 2005 & 24.405 .518 & 4.453 .156 & 19,24 \\
\hline 2006 & 24.285 .000 & 4.676 .646 & \\
\hline
\end{tabular}

Fonte: Gomes e Morais, 2007

Baseado nos dados do tamanho do sistema pode-se afirmar que o sistema de educação superior no Brasil pode ser caracterizado como sistema de elite até o ano de 2002, porque até então a população matriculada nunca foi superior a 15\%. É somente em 2003 que o volume de matrículas atinge a casa dos $16 \%$.

Embora o cenário desenhado nas afirmações supracitadas pareça ser aparentemente atraente para as instituições de ensino superior, não é o que vem acontecendo. No relatório de 2010 a Estácio afirma que perdeu 6,9\% de seus alunos. (ESTÁCIO DE SÁ, 2010). Além disto, segundo o Instituto Nacional de Estudos e Pesquisas (Inep) no Censo da Educação Superior 49,6\% das vagas oferecidas no ensino superior em 2008 não foram preenchidas. Isso significa que, somando a oferta em instituições públicas e particulares, das 3 milhões de vagas disponíveis, 1,47 milhão ficou completamente ociosa no ano passado. Comparando com 2007, o crescimento total das vagas não preenchidas foi de 11,6\%. 0 aumento mais expressivo ocorreu na rede federal, em que o número de vagas ociosas subiu $117 \%$. Nas universidades estaduais, o crescimento foi de $9 \%$, enquanto nas municipais, $6,9 \%$, e nas privadas, $10 \%$.

Além do crescimento de vagas ociosas, outra informação que identifica o acirramento do setor educacional é a quantidade de instituições que operam no Brasil.

Quadro 3: Evolução do número de instituições de ensino superior privadas - Brasil - 2000 a 2005

\begin{tabular}{|c|c|c|c|c|c|c|}
\hline IES & 2000 & 2001 & 2002 & 2003 & 2004 & 2005 \\
\hline IES privadas & 1.004 & 1.208 & 1.442 & 1.652 & 1.801 & 2.074 \\
\hline Total de IES & 1.180 & 1.391 & 1.637 & 1.859 & 2.020 & 2.310 \\
\hline
\end{tabular}

Fonte: Inep, 2009.

Em 2010, no site do e-mec, são 2.538 instituições de ensino superior credenciadas para operar no Brasil.

Observa-se em no ano de 2000 haviam 2.694.245 alunos matriculados, já em 2005 foram 4.453.156, o que corresponde um crescimento de 65,28\%. Já a quantidade de instituições passou de 1.180 para 2.310 , o que representa um crescimento de $96,76 \%$. Isto sem levar em consideração neste cálculo os novos campi que instituições já credenciadas abriram neste 
período. Isto mostra que mesmo crescendo o número de matriculados, houve um crescimento ainda maior no número de IES. Neste cenário, o Orçamento Base Zero poderia ser utilizado pelas instituições para conseguir manter-se operando neste mercado altamente competitivo.

\section{CONCLUSÃo}

O Orçamento Base Zero tornou-se opção de inúmeras empresas na tentativa de melhorar seu processo orçamentário em virtude das condições da atividade empresarial em constantes mudanças.

Diante da necessidade de melhorar o desempenho, principalmente neste cenário cada vez mais competitivo da educação superior no Brasil, seria aceitável que diversas instituições procurassem alterar seu elemento central do sistema de gestão: o orçamento. 0 Orçamento Base Zero torna-se uma opção para inúmeras empresas na tentativa de melhorar seu processo orçamentário. Porém, verificou-se que nos dados disponíveis em seus relatórios, nenhuma das instituições estudadas mencionou a utilização da ferramenta.

Considerando o objetivo da pesquisa de analisar a aplicação orçamentária nas instituições de ensino superior de Santa Cataria, focando na utilização do Orçamento Base Zero, este foi parcialmente alcançado. Isto porque apenas seis universidades puderam ser alvo mais aprofundado deste estudo, considerando que apenas estas tiveram documentos publicados em seus sites de relevância para o estudo. Foram elas: Unisul, UnC, Uniplac, Unesa, UFSC e Unp. Nos relatórios destas seis instituições estudadas, nenhum mencionou o Orçamento Base Zero como ferramenta utilizada ou a ser implantada na instituição.

Cabe ressaltar que a pesquisa teve a limitação de se basear em relatórios obrigatórios disponibilizados pelas instituições. Em um segundo momento uma entrevista com os controllers das instituições poderiam gerar uma corroboração da não utilização do Orçamento Base Zero para a elaboração orçamentária das instituições e posterior tomada de decisão organizacional.

No atual cenário educacional brasileiro, seria aconselhável a utilização do Orçamento Base Zero para as instituições de ensino superior considerando-se que o OBZ baseia-se em objetivos a longo prazo, metas específicas de curto prazo e os recursos financeiros disponíveis para ser bem sucedida, além de se concentrar nas atividades de despesas ao invés de departamento ou o tipo de item orçado que auxilia a controlar os gastos, pois o orçamento é montado a partir de zero em vez de ser construído sobre valores que foram gastos durante o período anterior, já que, na maioria das vezes, esses números já estão ultrapassados e possivelmente distorcidos.

\section{BIBLIOGRAFIA}

AKTEN, Mahmut; GIORDANO, Massimo; SCHEIFFELE, Mari A. Just-in-time budgeting for a volatile economy. McKinsey Quarterly; p115-121, 2009.

ALMEIDA, Lauro Brito de Almeida; MACHADO, Esmael Almeida Machado; RAIFUR, Léo; NOGUEIRA, Daniel Ramos. Apoio à Formulação de Estratégia, de Controle e de interatividade: Um Estudo Exploratório nas Cooperativas Agropecuárias da Região Sul do Brasil. Revista Contabilidade Vista \& Revista, Belo Horizonte, v. 20, n. 3, p. 65-99, jul./set. 2009.

AMARAL, N. C. Financiamento da educação superior: Estado x Mercado. São Paulo: Cortez, 
2003.

BATISTA, Andreia de Ávila. O Orçamento Base Zero (OBZ), O Controle de Custos e a Redução de Despesas nas Empresas: Agro Industrial Yamakawa e Agro Industrial Nova Andradina. Revista Raízes e Amidos Tropicais, v.3. Unesp: Botucatu, 2007.

BENNOUNA, Karim; MEREDITH, Geoffrey G.; MARCHANT, Teresa. Improved capital budgeting decision making: evidence from Canada.. Management Decision, Vol. 48, p225- 247, 2010.

COLOSSI, N. La universidad en el contexto de las transformaciones globales. In: COLÓQUIO INTERNACIONAL SOBRE GESTÃO UNIVERSITÁRIA NA AMÉRICA DO SUL, 2. Mar del Plata, 1993. Anais... Mar del Plata/Argentina: Universidad de Mar del Plata, 2001.

COSTA; MORITZ, MACHADO. Contribuições do Orçamento Base Zero no planejamento e controle de resultados em organizações empresariais. Revista Contemporânea de Contabilidade. Ano 4, vol. 1, n.8, p.85-98, jul./dez. 2007.

CHURCHILL, G.A. Marketing research: methodological foundations. Chicago: The Dryden Press, 1987.

E-MEC, Universidade Credenciadas para atuar em Santa Catarina. Disponível em: <http://emec.mec.gov.br/> Acesso em: 05 de abr. de 2009.

ESTÀCIO DE SÁ. Relatório de Gestão 2009. Disponível em: <http://www.estacioparticipacoes.com.br/estacio2010/web/arquivos/Estacio_DFP_2009.pd f>. Acesso em: 07 de abr. de 2010.

GOMES, Alfredo Macedo; MORAES, Karine Nunes de. A Expansão da Educação Superior no Brasil Contemporâneo: Questões para o Debate. AMPED, 2007.

INEP, Censo da Educação Superior. 2009. Disponível em: <http://www.inep.gov.br/imprensa/noticias/censo/superior/news09_05.htm>. Acesso em: 05 de abr. de 2010.

KING, Robyn; CLARKSON, Peter M.; WALLACE, Sandra. Budgeting practices and performance in small healthcare businesses. Management Accounting Research, Vol. 21, p40-55, Mar. 2010.

LAI, Joseph Hung Kit. Operation and maintenance budgeting for commercial buildings in Hong Kong. Construction Management \& Economics, Vol. 28, p415-427, Apr. 2010

LEITE, Rita Mara; SILVA, Helena de Fátima Nunes; CHEROBIM, Ana Paula Mussi Szabo; BUFREM, Leilah Santiago. Orçamento Empresarial: Levantamento da Produção Científica no Período de 1995 a 2006. Revista Contabilidade e Finanças. v. 19, n. 47, p. 56 - 72, maio/agosto 2008.

LUNKES, Rogério João. Orçamento de Base Zero - OBZ. Revista Brasileira de Contabilidade, v. 1, n. XXXII, p. 53-65, 2003.

MATURI, Richard J. Zero-Based Budgeting. Home Business Magazine: The Home-Based Entrepreneur's Magazine; Vol. 16, p70-70, May/Jun2009.

MELO, P. A. de. A Cooperação Universidade/Empresa nas universidades públicas 
brasileiras, Tese (Doutorado) - Universidade Federal de Santa Catarina, 2002.

MORGAN, B. F. Universidade de Brasília. A determinação do custo do ensino na educação superior: o caso da universidade de Brasília. Dissertação (Mestrado) - Universidade de Brasília, 2003.

PREMCHAND, A; ANTONAYA, A. Aspectos del Presupuesto Público. Fundo Monetário Internacional-FMI. Washington DC. 1988.

RAZA, Cláudio. Orçamento Base Zero: Modismo ou Necessidade. Revista Contábil \& Empresarial. Fiscolegis: Aracaju. Abril de 2010.

TEIXEIRA, A. Funções da universidade. Boletim Informativo CAPES. Rio de Janeiro, n.135, Fev. 1964.

TRIVIÑOS, A.N.S. Introdução à pesquisa em ciências sociais: a pesquisa qualitativa em educação. São Paulo: Atlas, 1994. 